Check for updates

Cite this: RSC Adv., 2017, 7, 41136

Received 19th June 2017

Accepted 17th August 2017

DOI: 10.1039/c7ra06816f

rsc.li/rsc-advances

\section{Encapsulation of transition metal dichalcogenides crystals with room temperature plasma deposited carbonaceous films $\dagger$}

\author{
Rakesh D. Mahyavanshi, (D) *a Golap Kalita, ${ }^{\star a}$ Rupesh Singh, ${ }^{c}$ Masaharu Kondo, ${ }^{\text {b }}$ \\ Takehisa Dewa, ${ }^{b}$ Toshio Kawahara, ${ }^{c}$ Masayoshi Umeno ${ }^{c}$ and Masaki Tanemura ${ }^{a}$
}

Encapsulation and its effect on two-dimensional (2D) dichalcogenides layered materials are of significant importance for electronic device fabrication. Here, we developed a dry and rapid encapsulation method for the $2 \mathrm{D}$ dichalcogenides by coating a carbonaceous film at room temperature using a plasma technique. Thin encapsulation film is coated on dichalcogenide crystals by microwave assisted surface wave plasma (MW-SWP) chemical vapor deposition (CVD) within a very short duration. Photoluminescence (PL) analysis shows the influence on excitation as well as the emission light with the coating of a carbonaceous film. The encapsulated dichalcogenide samples remain intact after heating at $200{ }^{\circ} \mathrm{C}$ as observed by $\mathrm{PL}$ and microscopic analysis. We also demonstrate obtaining stable photoconductivity for the dichalcogenide layers with encapsulation of a carbonaceous film. The rapid and dry coating technique at room temperature can be extended to various other 2D materials as the MW-SWP process allows damage-free encapsulation.

\section{Introduction}

In recent years, transition metal dichalcogenides (TMDCs) and other two-dimensional (2D) layered materials have attracted significant interest for nanoelectronic and optoelectronic device applications. ${ }^{1-5}$ The TMDCs are a large family of semiconductors (e.g. $\mathrm{MoS}_{2}, \mathrm{WS}_{2}$ etc.), metals (e.g. $\mathrm{TiS}_{2}, \mathrm{VSe}_{2}$ ), superconductors (e.g. $\left.\mathrm{TaS}_{2}, \mathrm{NbSe}_{2}\right)$, semimetals (e.g. $\mathrm{WTe}_{2}$ and $\mathrm{TiSe}_{2}$ ) and insulators (e.g. $\left.\mathrm{HfS}_{2}\right){ }^{6-16}$ The thickness dependent electronic and optoelectronic properties of group VI dichalcogenides $\left(\mathrm{MX}_{2}, \mathrm{M}=\mathrm{Mo}, \mathrm{W} ; \mathrm{X}=\mathrm{S}\right.$, Se, etc.) semiconductors are quite promising for novel optoelectronic devices. ${ }^{17,18}$ Transition from indirect band gap to direct band gap for the semiconducting trigonal prismatic $\mathrm{MX}_{2}$ has been revealed as the thickness reduces to monolayer. ${ }^{19-22}$ The $\mathrm{MX}_{2}$ semiconductor with excellent optical absorption in visible range and large exciton binding energy has attracted wide attention for highperformance photodetectors, efficient solar cells, and multifunctional nanoelectronics. ${ }^{23-26}$ TMDCs layered materials have been derived by exfoliation process, hydrothermal synthesis

${ }^{a}$ Department of Physical Science and Engineering, Nagoya Institute of Technology, Gokiso-cho, Showa-ku, Nagoya 466-8555, Japan. E-mail: rmahyavanshi@gmail.com; kalita.golap@nitech.ac.jp; Fax: +81-527355216; Tel: +81-527355216

${ }^{b}$ Department of Life Science and Applied Chemistry, Nagoya Institute of Technology, Gokiso-cho, Showa-ku, Nagoya 466-8555, Japan

'Department of Electronics and Information Engineering, Chubu University, 1200 Matsumoto-cho, Kasugai, 487-8501, Japan

$\dagger$ Electronic supplementary information (ESI) available. See DOI: $10.1039 / \mathrm{c} 7 \mathrm{ra06816f}$ and chemical vapor deposition (CVD) techniques. ${ }^{27-30}$ The CVD technique has a significant advantage for the synthesis of largearea polycrystalline films, single crystal domains, vertical or lateral heterostructures of two different dichalcogenides. ${ }^{29-32}$

Recent studies show structural transformation and degradation of $\mathrm{MX}_{2}$ layered materials with oxygen exposure, UV irradiation, ozone exposure and heating under ambient conditions. ${ }^{33-36}$ Significant structural degradation of $\mathrm{MX}_{2}$ layers has been also observed with long-term exposure to ambient conditions. ${ }^{36}$ The other 2D layered semiconductors, such as black phosphorus are more unstable and degradable under ambient conditions. ${ }^{37}$ In this context, encapsulation and device engineering are the key aspects that need to be addressed in the coming years for practical application of these layered semiconductors. Gao et al., has demonstrated coating of poly(methyl methacrylate) (PMMA) and Parylene C on $\mathrm{MoS}_{2}$ and $\mathrm{WS}_{2}$ layers to protect from ageing. ${ }^{36}$ Ahn et al. has demonstrated the transferring process of $\mathrm{PMMA} / \mathrm{h}-\mathrm{BN}$ layer on $\mathrm{WSe}_{2}$ layer as degradation protective layer. ${ }^{38}$ Recently, Woodhead et al. have developed an optical solution to encapsulate monolayer $\mathrm{MX}_{2}$ from physical damage and degradation. ${ }^{39}$ It has been also reported that depending on the layered materials a compressive or other lattice strain can be observed with the encapsulation layer. ${ }^{39,40}$ In contrast to the previous reports, we developed a rapid and dry approach to fully encapsulate $\mathrm{MX}_{2}$ layered materials preventing degradation in ambient conditions. As follows, we demonstrate the encapsulation and PL emission properties with the plasma CVD deposited carbonaceous film on $\mathrm{MX}_{2}$ layers. 


\section{Experimental section}

\subsection{Synthesis of $\mathrm{MoS}_{2}$ and $\mathrm{WS}_{2}$ crystals}

$\mathrm{MoS}_{2}$ and $\mathrm{WS}_{2}$ crystals were grown on $\mathrm{SiO}_{2} / \mathrm{Si}$ substrate by thermal chemical vapor deposition (CVD) method by controlling the growth parameters. The synthesis was performed in a quartz tube CVD system with a mixture of argon (Ar) and $\mathrm{H}_{2}$ gas flow at atmospheric pressure. Prior to the thermal CVD growth, the substrate was cleaned using acetone and following isopropyl alcohol (IPA) for $15 \mathrm{~min}$ each by sonication. Sulfur powder $(1 \mathrm{~g})$ in a ceramic boat was inserted into the quartz tube of length $90 \mathrm{~cm}$ and diameter of $4.5 \mathrm{~cm}$. It was placed in a low-temperature furnace (LTF) heated to $180{ }^{\circ} \mathrm{C}$. The substrate and $\mathrm{MoO}_{3}$ and $\mathrm{WO}_{3}$ powder $(10 \mathrm{mg})$ were kept on a ceramic boat for the growth of $\mathrm{MoS}_{2}$ and $\mathrm{WS}_{2}$ crystals, respectively in two separate experiments. $\mathrm{MoS}_{2}$ and $\mathrm{WS}_{2}$ crystals were synthesized at 750 and $850^{\circ} \mathrm{C}$, respectively in the high-temperature furnace (HTF). Both the experiments were carried out using 100 standard cubic centimeters per minute $(\mathrm{sccm})$ of argon and $\mathrm{H}_{2}$ gas mixture $(97: 3)$.

\subsection{Deposition of a carbonaceous film by plasma CVD}

Carbonaceous film was deposited by microwave assisted surface wave plasma (MW-SWP) chemical vapor deposition (CVD) technique on the $\mathrm{MoS}_{2}$ and $\mathrm{WS}_{2}$ crystals synthesized on $\mathrm{SiO}_{2} / \mathrm{Si}$ substrates. A metal grid was placed above the $\mathrm{MoS}_{2}$ and $\mathrm{WS}_{2}$ crystal samples (Fig. S1 $\dagger$ ) to avoid plasma induced deformation of the TMDCs layers. The MW-SWP CVD chamber was evacuated to $5 \times 10^{-4} \mathrm{~Pa}$ prior to the deposition. The thin film deposition was performed at 10-20 Pa gas composition pressure with a flow rate of 100-200 sccm of Ar and 10-50 sccm of $\mathrm{CH}_{4}$. The microwave power was fixed as $800 \mathrm{~W}$ with negligible amount of fluctuation. Deposition of the thin films was carried out for a duration of $60-240 \mathrm{~s}$ at room temperature $\left(\sim 24{ }^{\circ} \mathrm{C}\right)$.

\subsection{Characterization of prepared samples}

Prepared samples were characterized by optical microscopy, Raman spectroscopy, field-emission scanning electron microscopy (FE-SEM), photoluminescence (PL) spectroscopy and X-ray photoelectron spectroscopy (XPS). Optical microscopy analysis was performed using the digital optical microscope VHX-500 in reflectance mode with a Moticam 2000 2.0 M pixel camera. Raman analysis was carried out using the NRS 3300 laser Raman spectrometer with a laser excitation wavelength of $532.08 \mathrm{~nm}$. FE-SEM study was performed with JEOL JSM-7800F with an accelerating voltage of $5 \mathrm{kV}$. Infrared (IR) spectroscopy analysis was carried out with grazing incident using JASCO IRT-5000 FT-IR microscope. Absorbance and transmittance of the carbonaceous film was measured by JASCO V-670K spectrophotometer. XPS analysis was performed by VersaProbe using monochromated $\mathrm{Al} \mathrm{K \alpha}$ excitation source (1486.6 eV). PL analysis was carried with a confocal laser scanning microscope (NX-3DFLIM-N03, Tokyo Instruments, Japan) equipped with a $\mathrm{Nd}: \mathrm{YVO}_{4}$ diode laser. For the device fabrication, $\mathrm{Au}$ electrodes were deposited by the
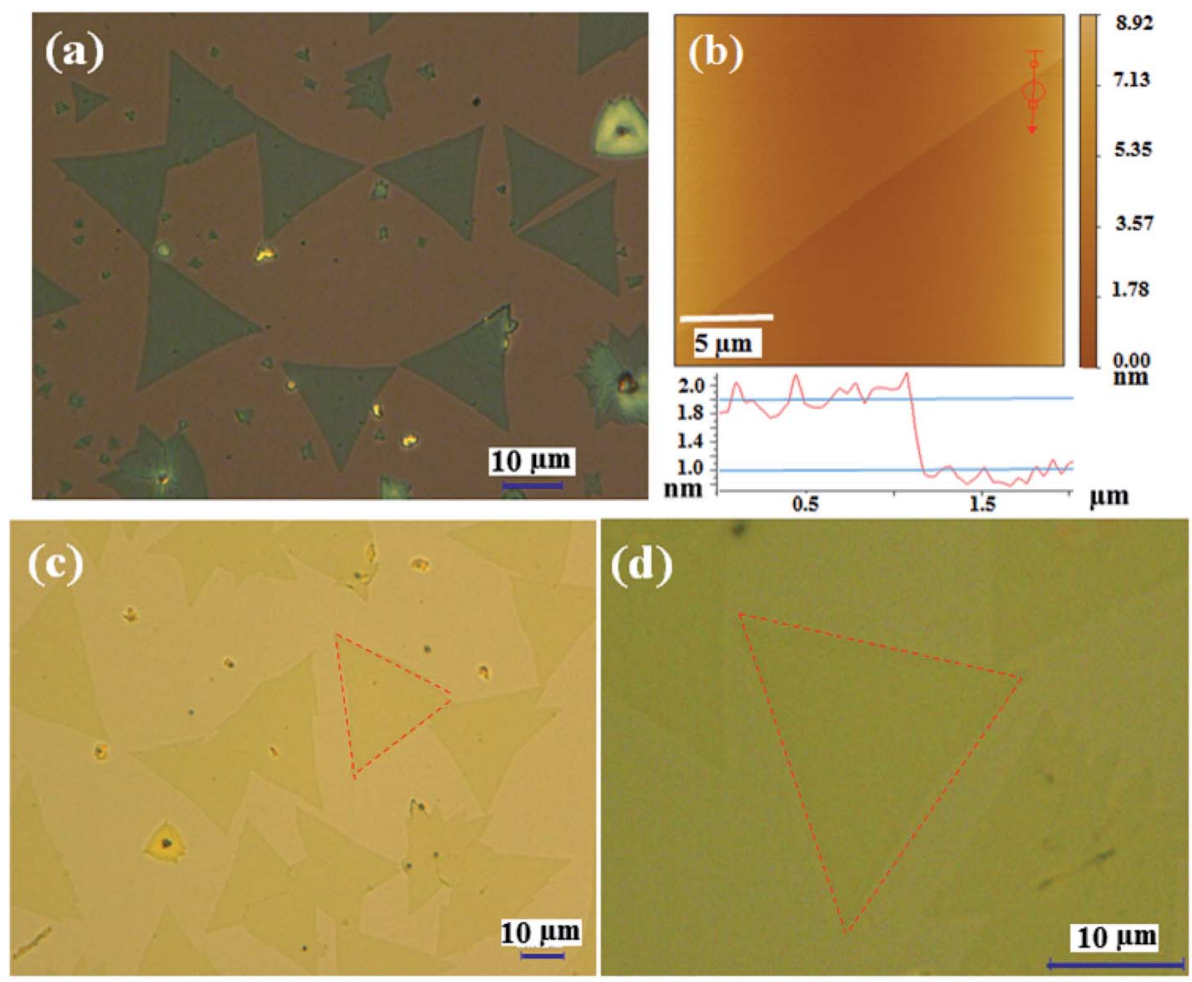

Fig. 1 (a) Optical microscope images of as-synthesized $\mathrm{MoS}_{2}$ crystals on the $\mathrm{SiO}_{2} / \mathrm{Si}$ substrate. (b) AFM image of $\mathrm{MoS}_{2}$ crystal and line profile presenting the thickness. Optical microscope images of carbonaceous film coated (c) $\mathrm{MoS}_{2} \mathrm{crystals}$ and (d) higher resolution view of a triangular $\mathrm{MoS}_{2}$ crystal. 
thermal evaporation technique using ULVAC VPC-260F. The current-voltage $(I-V)$ characteristics were measured using a two probe system and a Keithley 2401 SourceMeter.

\section{Results and discussion}

Fig. 1a shows optical microscope image of $\mathrm{MoS}_{2}$ crystal synthesized on the $\mathrm{SiO}_{2} / \mathrm{Si}$ substrate by thermal CVD technique. Triangular shaped $\mathrm{MoS}_{2}$ crystals with curved edges and size of around $20 \mu \mathrm{m}$ were obtained in the particular growth condition. Fig. 1b shows an AFM image of the as-synthesized $\mathrm{MoS}_{2}$ crystal. The line profile shows a thickness of nearly $\sim 0.9 \mathrm{~nm}$ for the individual $\mathrm{MoS}_{2}$ crystals. The synthesized samples were transferred to MW-SWP CVD chambers for coating without any other treatment. Microwave excited plasma sources have been used for effective deposition of an insulating encapsulation layer for the conventional semiconductors. ${ }^{\mathbf{4 1 , 4 2}}$ We explore the possibility of 2D layered materials encapsulation at room temperature by SW-MWP technique. The MW-SWP can produce an over-dense plasma even at a low gas pressure, with a density higher than the cut-off density $\left(7.4 \times 10^{16} \mathrm{~m}^{-3}\right.$ for $\left.2.45 \mathrm{GHz}\right)$. The deposition process was rapid and substrates were place at a longer distance from plasma shower which allowed to maintain the substrate temperature at $\sim 24{ }^{\circ} \mathrm{C}$. Fig. 1c shows an optical microscope image of the coated $\mathrm{MoS}_{2}$ crystals. The triangular $\mathrm{MoS}_{2}$ crystal remain intact without any visible structural damage. Fig. 1d shows a higher resolution optical microscopy image of the $\mathrm{MoS}_{2}$ triangular crystals, where the morphology is unaffected by deposition of the encapsulation film. Unlike other plasma technique, the MW-SWP is a promising tool for large-area carbonaceous film deposition and useful to avoid plasma induced damages of the substrate surface. ${ }^{43}$ The details of the deposition process and photograph of the deposited film are included in ESI (Fig. S1 and S2 $\dagger$ ).

Fig. 2a shows an IR absorption spectra of the carbonaceous film coated on the $\mathrm{MoS}_{2} / \mathrm{SiO}_{2} / \mathrm{Si}$ sample $(\sim 15 \mathrm{~nm})$. Few weak peaks are observed in the frequency range from 1300 to $1600 \mathrm{~cm}^{-1}$, where the peak centered at $\sim 1530 \mathrm{~cm}^{-1}$ can be assigned to $\mathrm{C}=\mathrm{C}$ stretching vibrations. At a higher frequency range, three sharper peaks at 2870, 2925 and $2961 \mathrm{~cm}^{-1}$ were observed corresponding to the $\mathrm{C}-\mathrm{H}$ vibrations. These absorption peaks can be assigned to $\mathrm{sp}^{3} \mathrm{CH}_{x}$ symmetric and asymmetric bands. Fig. 2b shows a typical Raman spectra of the carbonaceous coating. There was no significant Raman features for the plasma deposited carbonaceous film, other than a broad hump and reduction in intensity of Si Raman peak. A carbon film coated around $\sim 150{ }^{\circ} \mathrm{C}$ with similar plasma parameters shows broad $\mathrm{D}$ and $\mathrm{G}$ peaks, clearly indicating the difference in
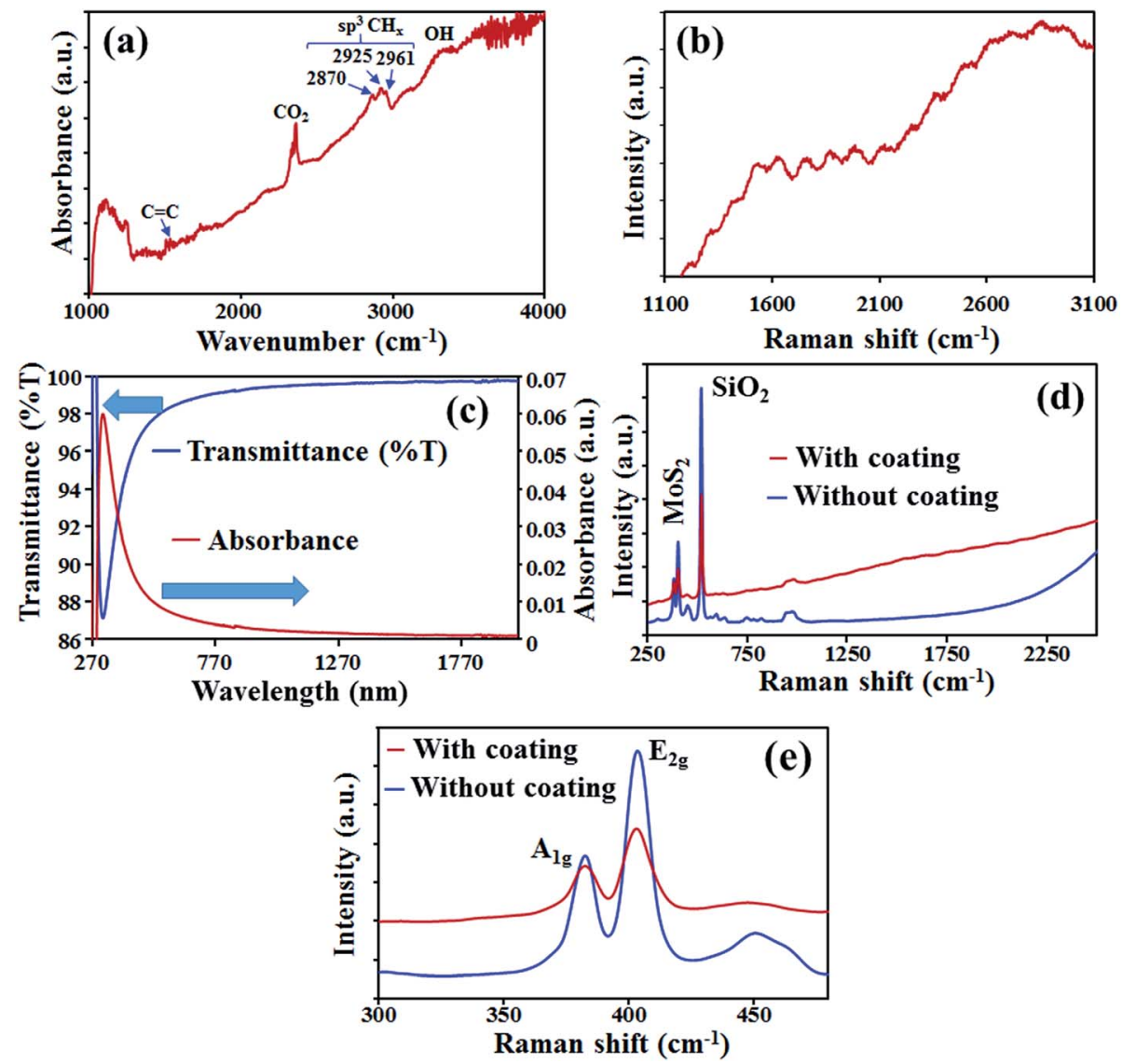

Fig. 2 (a) IR (b) Raman (c) transmittance and absorbance spectra of the carbonaceous film on $\mathrm{SiO}_{2} / \mathrm{Si}$ and glass substrates, deposited along with the coating of TMDCs crystals samples. Raman spectra of the (d) $\mathrm{MoS}_{2}$ crystal on $\mathrm{SiO}_{2} / \mathrm{Si}$ substrate (e) $\mathrm{A}_{1 \mathrm{~g}}$ and $\mathrm{E}_{2 \mathrm{~g}}$ peaks with and without encapsulation film. 
carbon structures for room temperature deposited film (Fig. S3†). The presence of $\mathrm{sp}^{3} \mathrm{CH}_{x}$ bonds and no clear Raman peaks ( $D$ and $G$ bands) signifies formation of insulating carbonaceous film with polymeric nature. ${ }^{43,44}$ The room temperature deposited carbon film is significantly different than that of normal crystalline and amorphous carbon forms. Fig. 2c shows transmittance and absorbance of the carbonaceous film deposited on glass substrate. The coated film shows excellent transparency in the wavelength range of $0.3-2 \mu \mathrm{m}$. More than $95 \%$ transparency is obtained in the visible light region, which can be tuned with appropriate thickness of the deposited film. The coated film on $\mathrm{SiO}_{2} / \mathrm{Si}$ substrate was characterized by Raman spectroscopy with and without the TMDCs crystals. Fig. $2 \mathrm{~d}$ and e shows Raman spectra of the $\mathrm{MoS}_{2}$ crystals with and without encapsulated film. The Raman vibration modes $\mathrm{E}_{2 \mathrm{~g}}^{1}$ and $\mathrm{A}_{1 \mathrm{~g}}$ for $\mathrm{MoS}_{2}$ are observed at 383.2 and 403.3 $\mathrm{cm}^{-1}$, (frequency difference of $\sim 20 \mathrm{~cm}^{-1}$ ) corresponding to inplane vibration of molybdenum and sulfur and out-of-plane vibration of sulfur atoms, respectively. We observed a reduction in Raman peak intensity for the encapsulated sample, while no significant shift in peak position. Influence of the coated layer was further analyzed by PL study to investigate the possible effect on PL excitation and light emission.

Fig. 3a shows FE-SEM image of the as-synthesized $\mathrm{MoS}_{2}$ crystals on $\mathrm{SiO}_{2} / \mathrm{Si}$ substrate by the CVD technique. The triangular shaped $\mathrm{MoS}_{2}$ crystals can be confirmed by the FE-SEM analysis. Fig. 3b shows the FE-SEM image of the plasma deposited carbonaceous film on $\mathrm{MoS}_{2}$ crystals. The surface morphology of the encapsulated $\mathrm{MoS}_{2}$ sample was observed by FE-SEM analysis using the upper electron detector (UED) and $2 \mathrm{kV}$ accelerating voltage. The triangular $\mathrm{MoS}_{2}$ crystals can be confirmed below the encapsulation layer, although the nonconducting encapsulation film significantly affects the micrograph. Previously, plasma functionalization of TMDCs layer has been also demonstrated for modification of electronic properties. PL properties of the encapsulated $\mathrm{MoS}_{2}$ layer were investigated considering the surface functionalization by the plasma process and possible effect on PL emission. ${ }^{45}$ Fig. 3c shows the PL spectra of coated and non-coated $\mathrm{MoS}_{2}$ crystal. Quenching and red shift of the PL peak for the encapsulated $\mathrm{MoS}_{2}$ sample was observed (Fig. 3c). The PL quenching and shift confirm influence of PL excitation and emission of light for the $\mathrm{MoS}_{2}$ crystal with the encapsulation. Woodhead et al. have also reported significant suppression of the PL peak of $\mathrm{MoS}_{2}$ monolayer by solution coated epoxy due to the compressive strain..$^{39}$ Compressive strain can modulate the direct to indirect band gap of $\mathrm{MoS}_{2}$ crystals, which reduces the emission intensity. The required values of strain to induce a shift from direct to indirect bandgaps for $\mathrm{MoS}_{2}$ and $\mathrm{WS}_{2}$ are $0.5 \%$ and $1 \%$ respectively. ${ }^{22}$ However, the reduction and red shift of the PL peak with the carbonaceous encapsulated layer cannot solely confirm as compressive strain, which was further investigated for the triangular $\mathrm{WS}_{2}$ crystals.

Fig. 4a shows optical microscope image of CVD synthesized triangular $\mathrm{WS}_{2}$ crystals on $\mathrm{SiO}_{2} / \mathrm{Si}$ substrate. Fig. $4 \mathrm{~b}$ shows optical microscope image of $\mathrm{WS}_{2}$ crystals on the $\mathrm{SiO}_{2} / \mathrm{Si}$ substrate after coating a thin carbonaceous film $(\sim 15 \mathrm{~nm})$. Similarly, Fig. 4c shows an optical microscope image of $\mathrm{WS}_{2}$ sample with a thicker coated layer $(\sim 30 \mathrm{~nm})$. The difference in coating thickness can be also identified with variation in contrast of the optical images. The $\mathrm{WS}_{2}$ crystals remain unaffected after the room temperature deposited encapsulation
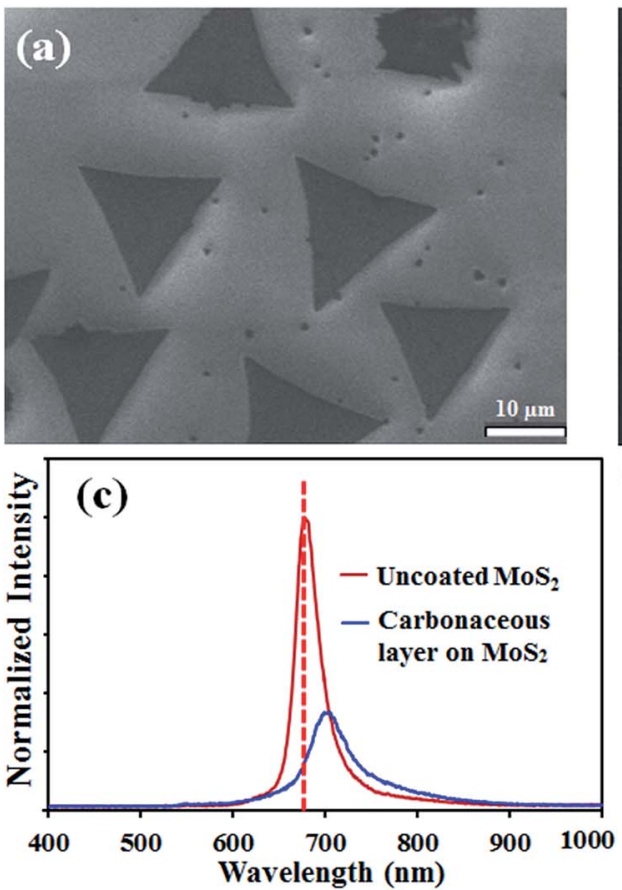

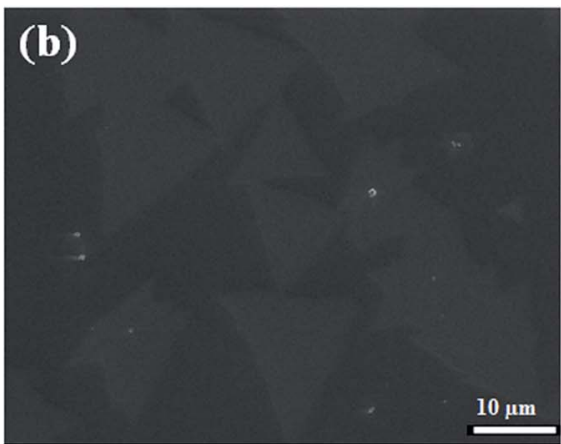

(d)

\section{Plasma deposited carbonaceous Layer on dichalcogenide crystals}

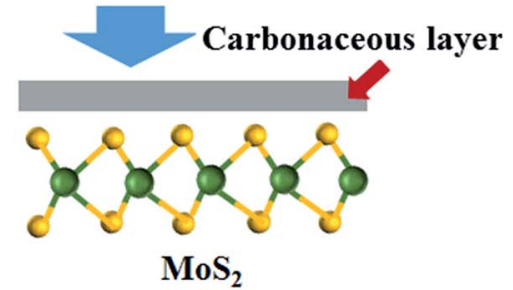

Fig. 3 FE-SEM image of (a) as-synthesized and (b) carbonaceous film coated $\mathrm{MoS}_{2}$ crystals. (c) Photoluminescence (PL) spectra of the uncoated and coated $\mathrm{MoS}_{2}$ crystal. (d) Schematic of plasma deposited carbonaceous film encapsulation on $\mathrm{MoS}_{2} \mathrm{Crystal}_{\text {. }}$ 

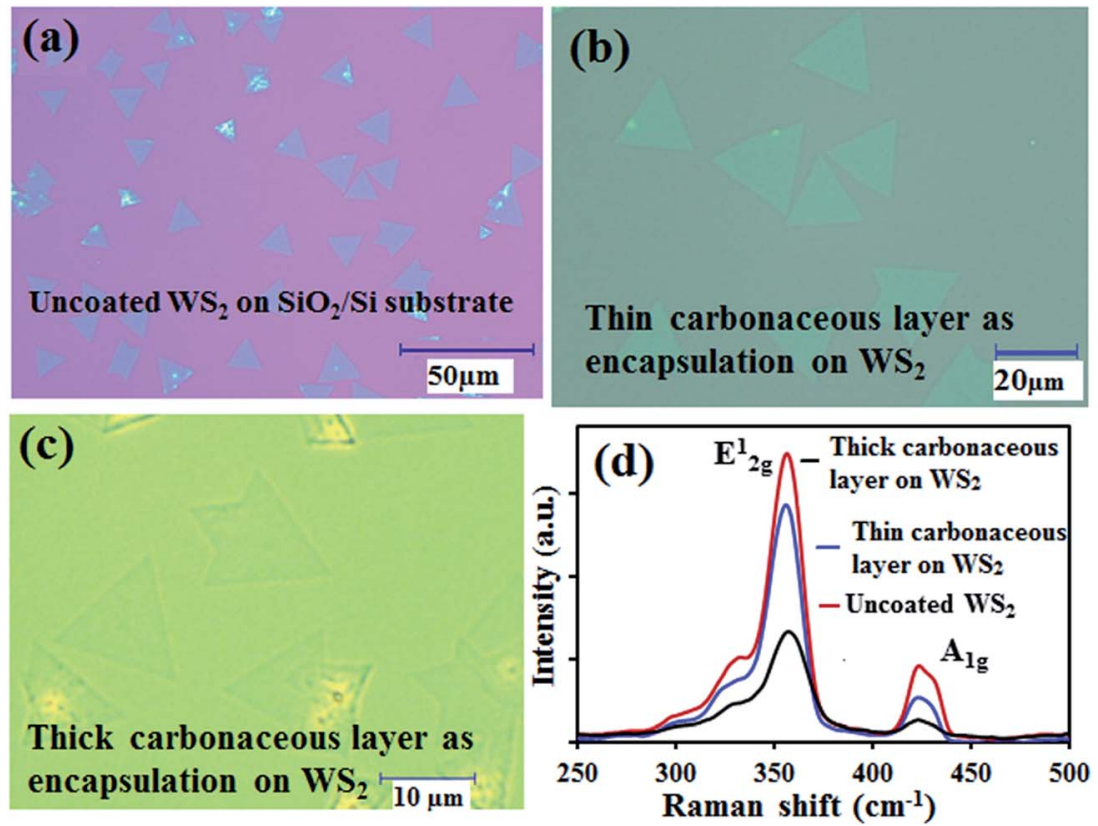

Fig. 4 Optical microscope image of (a) CVD synthesized triangular $\mathrm{WS}_{2}$ crystals on $\mathrm{SiO}_{2} / \mathrm{Si}$ substrate, (b) and (c) MW-SWP deposited carbonaceous film on the CVD synthesized WS $\mathrm{W}_{2}$ crystals with different thickness. (d) Raman spectra of the $\mathrm{WS}_{2} \mathrm{crystal}$ before and after encapsulation.

layer as observed from the optical microscopy images. Further, Raman and X-ray photoelectron spectroscopy (XPS) analysis were carried out to investigate the structural properties. XPS analysis of the as growth dichalcogenide layers and encapsulated samples are included in ESI (Fig. S4 and S5†). Fig. 4d shows the Raman spectra of the $\mathrm{WS}_{2}$ crystals before and after coating the encapsulation layer. The as-synthesized $\mathrm{WS}_{2}$ crystals on $\mathrm{SiO}_{2} / \mathrm{Si}$ substrate shows characteristic Raman shift at $\sim 356$ and $\sim 423 \mathrm{~cm}^{-1}$ corresponding to $\mathrm{E}_{2 \mathrm{~g}}$ mode for in-plane vibration of tungsten and sulfur atoms and $\mathrm{A}_{1 \mathrm{~g}}$ mode for out-of-plane vibration of sulfur atoms, respectively. Although, the Raman shift does not show any considerable change from the original sample, the intensity of Raman peak show reduction with increasing thickness of encapsulation layer. The structure and the chemical nature of the $\mathrm{WS}_{2}$ crystals is virtually unaffected by carbonaceous coating as the Raman vibration modes are at the same frequency for as synthesized as well as coated samples. Raman analysis also does not show substantial evidence of strain, while significant reduction and shift of PL peak were evident. ${ }^{36,39,46,47}$ Fangfei Li et al. has reported only small shift in Raman peaks with applying a significantly high pressure $(0.84 \mathrm{GPa})$ to induce a compressive strain. ${ }^{46}$

The encapsulation behavior of $\mathrm{WS}_{2}$ crystals with the plasma deposited carbonaceous film was investigated by accelerated heating test in atmospheric condition. Fig. 5a shows FE-SEM image of a pristine triangular $\mathrm{WS}_{2}$ crystals after heating in atmosphere at $200{ }^{\circ} \mathrm{C}$ for 1 hour. White traces and their branched structures appear thought-out the crystals, signifying the heat-induced degradation of the $\mathrm{MX}_{2}$ layer under atmospheric conditions. Fig. 5b shows FE-SEM image of the encapsulated $\mathrm{WS}_{2}$ crystals after the same accelerated heating test. The triangular $\mathrm{WS}_{2}$ crystals remain intact, however, it is difficult to identify any kind of traces as observed for the uncoated sample due to the presence of the encapsulation layer. PL analysis was carried out to investigate the encapsulation effect of the $\mathrm{WS}_{2}$ crystals before and after the heating of the samples. Fig. 5c shows PL spectra for the uncoated and encapsulated $\mathrm{WS}_{2}$ samples. The intensities were normalized for maximum value to obtain a clear idea of the relative changes in PL. A sharp PL peak at $655 \mathrm{~nm}(1.89 \mathrm{eV})$ was obtained for the pristine $\mathrm{WS}_{2}$ crystals. Significant difference in PL peak was observed with coating a carbonaceous layer on the $\mathrm{WS}_{2}$ crystals. Coating a thin encapsulation layer $(\sim 15 \mathrm{~nm})$ on the $\mathrm{WS}_{2}$ crystals, decrease in PL intensity and red shift (shifted to $662 \mathrm{~nm}$ corresponding to $1.87 \mathrm{eV}$ ) was observed (Fig. 5c). By increasing the thickness of the encapsulation layer $(\sim 30 \mathrm{~nm})$, we observed a much reduced PL peak with the similar red shift. It confirms the influence of the carbonaceous film thickness on the PL excitation as well as the emitted light. Then, PL analysis was performed for the coated and uncoated $\mathrm{WS}_{2}$ samples after heating at $200{ }^{\circ} \mathrm{C}$ in atmospheric condition. Fig. 5d shows the normalized PL spectra for the heating tested samples. After the heating process, both the encapsulated $\mathrm{WS}_{2}$ samples show similar intensity PL peaks with a peak position at $650 \mathrm{~nm}(1.90 \mathrm{eV})$. The PL peak of the uncoated $\mathrm{WS}_{2}$ crystals was reduced due to the degradation under atmospheric heating as observed from the SEM analysis. Interestingly, the PL peak position of the coated sample slightly blue shifted $(\sim 5 \mathrm{~nm})$ and significantly differed prior to the heating $(\sim 12 \mathrm{~nm})$. The effect is complicated to understand, as different process can occurs, such as reduction in internal absorption of emitted light and change in induced strain. ${ }^{48}$ Then, the coated samples were further tested by heating at a higher temperature $\left(250^{\circ} \mathrm{C}\right)$ in atmospheric condition. Fig. 5e shows the optical image of the carbonaceous coated $\mathrm{WS}_{2}$ 

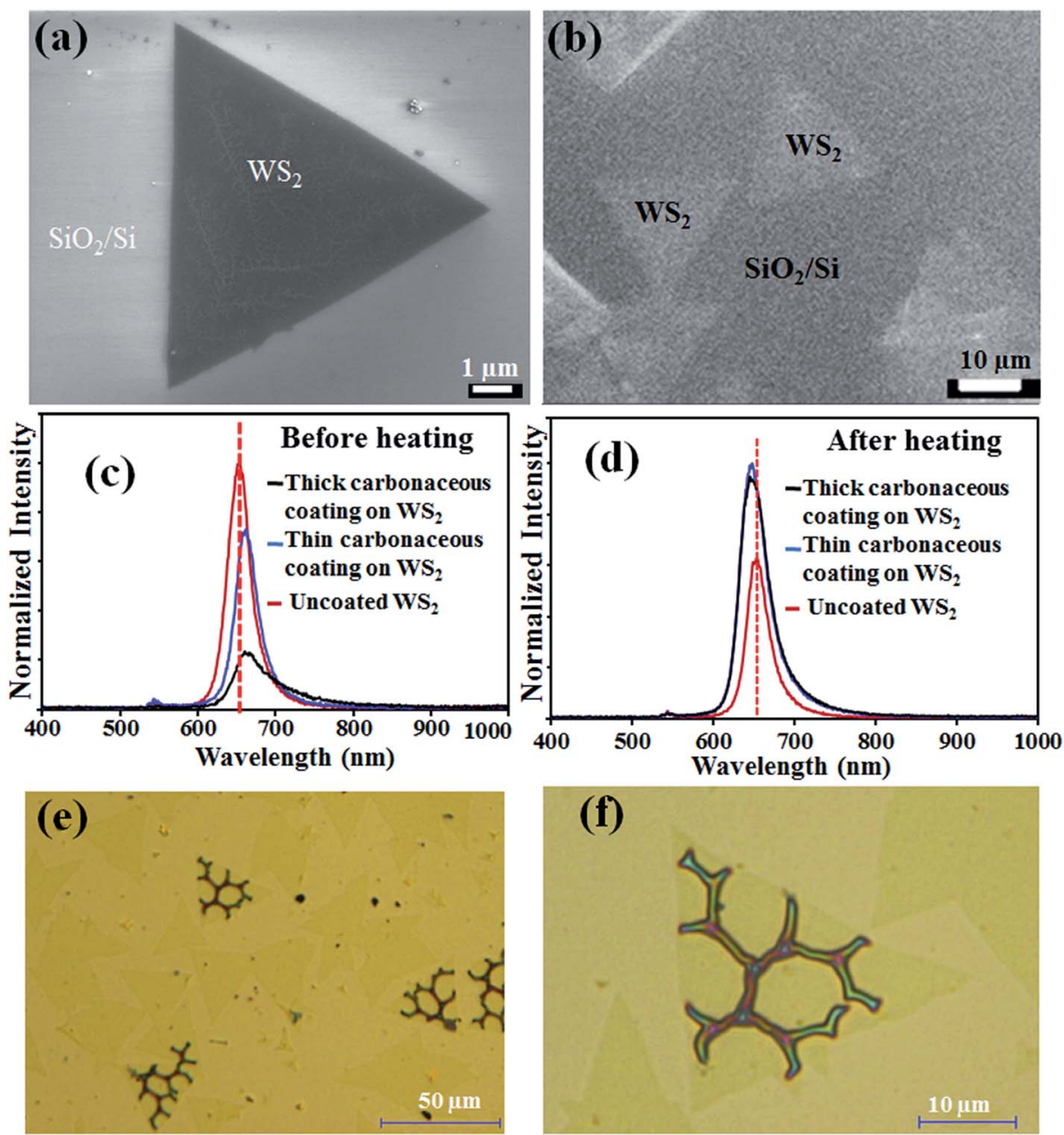

Fig. 5 FE-SEM images of triangular WS 2 crystals (a) without and (b) with coating a carbonaceous film after heating in atmosphere at $200{ }^{\circ} \mathrm{C}$ for 1 hour. PL spectra for (c) before and (d) after heating of the $\mathrm{WS}_{2}$ crystals with different thickness of encapsulation layers in comparison with uncoated sample. Optical microscope images of encapsulated (e) $W S_{2}$ sample and (f) cracks formation in a WS $\mathrm{S}_{2}$ crystals after heating in atmosphere at $250^{\circ} \mathrm{C}$.

sample after the heating experiment. We observed crack formation in some of the $\mathrm{WS}_{2}$ crystals after the heating process. Fig. 6d shows a higher magnified view of the coated $\mathrm{WS}_{2}$ crystals with clearly visible cracks. The cracks appeared in the $\mathrm{WS}_{2}$ crystals, which propagate in hexagonal patterns, with nearly $120^{\circ}$ angles. This signifies localized heat induced defect enhancement in the individual encapsulated $\mathrm{WS}_{2}$ crystals. ${ }^{36}$ Significantly, we observed the appearance of the cracks only in few $\mathrm{WS}_{2}$ crystals, where all other crystals remain unaffected. The reason behind this anomaly can be attributed to the difference in defect level of individual crystals and variation in induced strain with thermal expansion differences. It can be expected that better thermal conductivity of the TMDCs crystals will lead to relatively higher local temperature of $\mathrm{WS}_{2}$ than the other areas of carbonaceous encapsulated sample. Previously, Yan et al. has reported the local heat induced thermal expansion properties of the TMDCs layer. ${ }^{40}$

The effect of encapsulation layer on electrical and optical properties of $\mathrm{MoS}_{2}$ was studied by Schottky junction fabrication. Fig. 6a shows the schematic diagram of the $\mathrm{Au} / \mathrm{MoS}_{2} / \mathrm{Au}$ device with the carbonaceous layer. The distance between two $\mathrm{Au}$ electrodes was approximately $200 \mu \mathrm{m}$ covering $\mathrm{MoS}_{2}$ layer in between as shown in the optical microscope image (Fig. 6b). I-V characteristics of the device was measured with (white light, $\sim 100 \mathrm{~mW} \mathrm{~cm}{ }^{-2}$ ) and without light illumination conditions. Fig. 6c shows $I-V$ characteristic under dark and light conditions of the as-fabricated device with plasma deposited encapsulated layer. Nonlinear Schottky junction behavior was obtained for the semiconducting $\mathrm{MoS}_{2}$ layer and Au metal electrode, where the encapsulated layer did not show much influence on junction behavior. With illumination of light, we observed significant photo-response for Schottky junction with photo-exciton in the $\mathrm{MoS}_{2}$ layer. Then, the device was annealed at $200{ }^{\circ} \mathrm{C}$ under atmospheric condition for $1 \mathrm{~h}$ as discussed above. Fig. $6 \mathrm{~d}$ shows $I-V$ characteristic of the same device after annealing. The Schottky junction behavior and photo-responsivity was unchanged and no hysteresis was observed with the annealing in presence of the encapsulating layer. Thus, the effectiveness of carbonaceous encapsulating layer on electrical and photoresponsive properties of $\mathrm{MoS}_{2}$ layer was confirmed. 
(a)
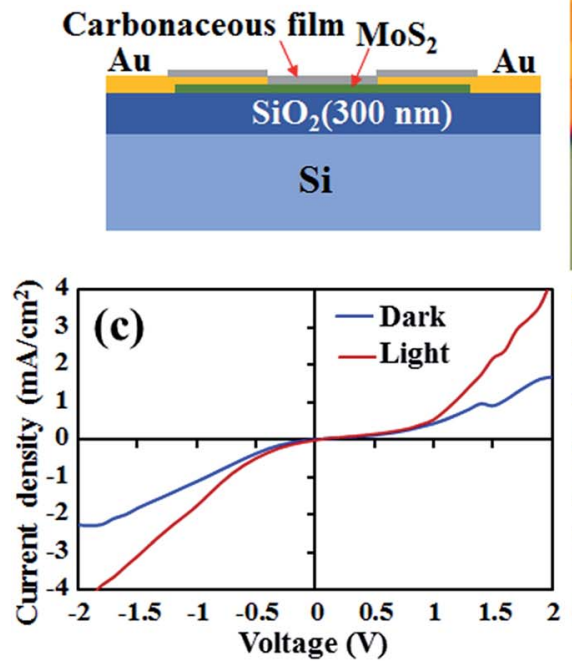
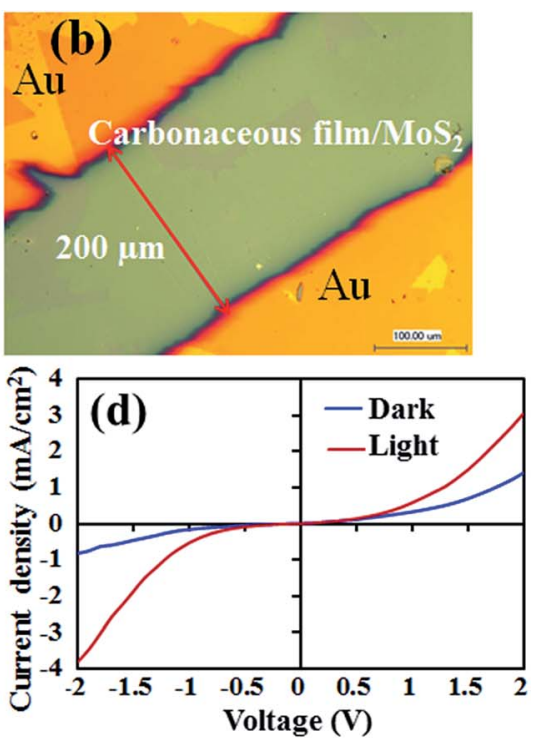

Fig. 6 (a) Schematic diagram and (b) optical microscope image of $\mathrm{Au} / \mathrm{MoS}_{2} / \mathrm{Au}$ device with encapsulating carbonaceous layer. Current-voltage

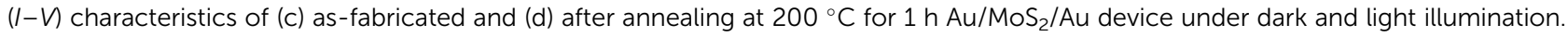

\section{Conclusion}

In conclusion, we have demonstrated deposition of a carbonaceous film at room temperature on $\mathrm{MoS}_{2}$ and $\mathrm{WS}_{2}$ crystals by the SW-MWP CVD technique as an encapsulation layer. The encapsulation process is dry, rapid and nondestructive, which can be carried out at room temperature for large area substrate. PL analysis showed the influence on excitation as well as the emission light with the coating of a carbonaceous film. The encapsulated samples remained intact as observed by the optical microscopy, SEM and PL analysis in an accelerated degradation test by heating in ambient condition (humidity $\sim 65 \%$ ). We also observed change in PL emission for the encapsulated $\mathrm{WS}_{2}$ layer after heating process, attributing to reduction in internal absorption of emitted light and change in induced strain. We have also demonstrated obtaining stable photoconductivity for the TMDCs dichalcogenide layers with encapsulation of a carbonaceous film. The rapid and dry coating technique at room temperature can be extended to various other $2 \mathrm{D}$ materials as MW-SWP process allows damage free encapsulation.

\section{Conflicts of interest}

There are no conflicts to declare.

\section{References}

1 K. F. Mak, C. Lee, J. Hone, J. Shan and T. F. Heinz, Phys. Rev. Lett., 2010, 105, 136805.

2 Q. H. Wang, K. Kalantar-Zadeh, A. Kis, J. N. Colema and M. S. Strano, Nat. Nanotechnol., 2012, 7, 699-712.
3 K. S. Novoselov, A. K. Geim, S. V. Morozov, D. Jiang, Y. Zhang, S. V. Dubonos, I. V. Grigorieva and A. A. Firsov, Science, 2004, 306, 666-669.

4 P. Vogt, P. De Padova, C. Quaresima, J. Avila, E. Frantzeskakis, M. C. Asensio, A. Resta and B. G. Le Lay, Phys. Rev. Lett., 2012, 108, 155501.

5 L. Li, Y. Yu, G. Jun Ye, Q. Ge, X. Ou, H. Wu, D. Feng, X. H. Chen and Y. Zhang, Nat. Nanotechnol., 2014, 9, 372377.

6 Z. Y. Zeng, C. L. Tan, X. Huang, S. Y. Bao and H. Zhang, Energy Environ. Sci., 2014, 7, 797-803.

7 F. Y. Li, K. X. Tu and Z. F. Chen, J. Phys. Chem. C, 2014, 118, 21264-21274.

8 L. Ma, C. Ye, Y. Yu, X. F. Lu, X. Niu, S. Kim, D. Feng, D. Tomanek, Y. W. Son, X. H. Chen and Y. Zhang, Nat. Commun., 2016, 7, 10956.

9 X. Xi, L. Zhao, Z. Wang, H. Berger, L. Forro, J. Shan and K. F. Mak, Nat. Nanotechnol., 2015, 10, 765-769.

10 M. M. Ugeda, A. J. Bradley, Y. Zhang, S. Onishi, Y. Chen, W. Ruan, C. Ojeda-Aristizabal, H. Ryu, M. T. Edmonds, H. Z. Tsai, A. Riss, S. K. Mo, D. H. Lee, A. Zettl, Z. Hussain, Z. X. Shen and M. F. Crommie, Nat. Phys., 2016, 12, 92-97.

11 T. R. Chang, S. Y. Xu, G. Chang, C. C. Lee, S. M. Huang, B. Wang, G. Bian, H. Zheng, D. S. Sanchez, I. Belopolski, et al., Nat. Commun., 2016, 7, 10639.

12 K. Sugawara, Y. Nakata, R. Shimizu, P. Han, T. Hitosugi, T. Sato and T. Takahashi, ACS Nano, 2016, 10, 1341-1345.

13 Y. I. Joe, X. M. Chen, P. Ghaemi, K. D. Finkelstein, G. A. de la Pena, Y. Gan, J. C. T. Lee, S. Yuan, J. Geck, G. J. MacDougall, et al., Nat. Phys., 2014, 10, 421-425.

14 B. Radisavljevic, A. Radenovic, J. Brivio, V. Giacometti and A. Kis, Nat. Nanotechnol., 2011, 6, 147-150. 
15 W. Yang, J. Shang, J. Wang, X. Shen, B. Cao, N. Peimyoo, C. Zou, Y. Chen, Y. Wang, C. Cong, et al., Nano Lett., 2016, 16, 1560-1567.

16 A. Thangaraja, S. M. Shinde, G. Kalita and M. Tanemura, Appl. Phys. Lett., 2016, 108, 053104.

17 K. F. Mak, K. He, J. Shan and T. F. Heinz, Nat. Nanotechnol., 2012, 7, 494-498.

18 J. K. Ellis, M. J. Lucero and G. E. Scuseria, Appl. Phys. Lett., 2011, 99, 261908.

19 T. Cheiwchanchamnangij and W. R. L. Lambrecht, Phys. Rev. B, 2012, 85, 205302.

20 A. Splendiani, L. Sun, Y. Zhang, T. Li, J. Kim, C. Y. Chim, G. Galli and F. Wang, Nano Lett., 2010, 10, 1271-1275.

21 M. Amani, D. H. Lien, D. Kiriya, J. Xiao, A. Azcatl, J. Noh, S. R. Madhvapathy, R. Addou, S. Kc, M. Dubey, et al., Science, 2015, 350, 1065-1068.

22 Z. Wang, Z. Dong, Y. Gu, Y. Chang, L. Zhang, L. Li, W. Zhao, G. Eda, W. Zhang, G. Grinblat, et al., Nat. Commun., 2016, 7, 11283.

23 R. Cheng, D. Li, H. Zhou, C. Wang, A. Yin, S. Jiang, Y. Liu, Y. Chen, Y. Huang and X. Duan, Nano Lett., 2014, 14, 5590-5597.

24 O. Lopez-Sanchez, D. Lembke, M. Kayci, A. Radenovic and A. Kis, Nat. Nanotechnol., 2013, 8, 497-501.

25 X. Hong, J. Kim, S. F. Shi, Y. Zhang, C. Jin, Y. Sun, S. Tongay, J. Wu, Y. Zhang and F. Wang, Nat. Nanotechnol., 2014, 9, 682-686.

26 M. L. Tsai, S. H. Su, J. K. Chang, D. S. Tsai, C. H. Chen, C. I. Wu, L. J. Li, L. J. Chen and J. H. He, ACS Nano, 2014, 8, 8317-8322.

27 G. Eda, H. Yamaguchi, D. Voiry, T. Fujita, M. Chen and M. Chhowalla, Nano Lett., 2011, 11, 5111-5116.

28 Q. Ji, Y. Zhang, Y. Zhang and Z. Liu, Chem. Soc. Rev., 2015, 44, 2587.

29 Y. Zhang, Y. Zhang, Q. Ji, J. Ju, H. Yuan, J. Shi, T. Gao, D. Ma, M. Liu, Y. Chen, et al., ACS Nano, 2013, 7, 8963-8971.

30 J. Yang, Y. Gu, E. Lee, H. Lee, S. H. Park, M. H. Cho, Y. H. Kim, Y. H. Kim and H. Kim, Nanoscale, 2015, 7, 9311-9319.

31 K. N. Kang, K. Godin and E. H. Yang, Sci. Rep., 2015, 5, 13205.
32 Y. Lee, X. Zhang, W. Zhang, M. Chang, C. Lin, K. Chang, Y. Yu, J. Wang, C. Chang, L. Li and T. Lin, Adv. Mater., 2012, 24, 2320-2325.

33 D. Mahalu, M. Peisach, W. Jaegermann, A. Wold and R. Tenne, J. Phys. Chem., 1990, 94, 8012-8013.

34 Y. Rong, K. He, M. Pacios, A. W. Robertson, H. Bhaskaran and J. H. Warner, ACS Nano, 2015, 9, 3695-3703.

35 Y. Liu, C. Tan, H. Chou, A. Nayak, D. Wu, R. Ghosh, H. Y. Chang, Y. Hao, X. Wang, J. Kim, et al., Nano Lett., 2015, 15, 4979-4984.

36 J. Gao, B. Li, J. Tan, P. Chow, T. M. Lu and N. Koratkar, ACS Nano, 2016, 10, 2628-2635.

37 J. D. Wood, S. A. Wells, D. Jariwala, K. S. Chen, E. Cho, V. K. Sangwan, X. Liu, L. J. Lauhon, T. J. Marks and M. C. Hersam, Nano Lett., 2014, 14, 6964-6970.

38 S. Ahn, G. Kim, P. K. Nayak, S. I. Yoon, H. Lim, H. J. Shin and H. S. Shin, ACS Nano, 2016, 10, 8973-8979.

39 C. S. Woodhead, J. Roberts, Y. J. Noori, Y. Cao, R. BernardoGavito, P. Tovee, A. Kozikov, K. Novoselov and R. J. Young, 2D Mater., 2017, 4, 015032.

40 R. Yan, S. Bertolazzi, J. Brivio, T. Fang, A. Konar, A. G. Birdwell, N. V. Nguyen, A. Kis, D. Jena and H. Xing, 2013, arXiv:1211.4136.

41 D. R. Cote, G. Y. Lee, S. V. Nguyen, A. K. Stamper, D. S. Armbrust, D. Tobben, R. A. Conti and G. Y. Lee, IBM J. Res. Dev., 1999, 43, 5-38.

42 S. Uenoa, Y. Konishi and K. Azuma, ECS J. Solid State Sci. Technol., 2016, 5, R21-R25.

43 J. Xiao, M. Yao, K. Zhu, D. Zhang, S. Zhao, S. Lu, B. Liu, W. Cui and B. Liu, Nanoscale, 2013, 5, 11306-11312.

44 G. Kalita, H. R. Aryal, S. Adhikari, D. C. Ghimire, R. A. Afre, T. Soga, M. Sharon and M. Umeno, Diamond Relat. Mater., 2008, 17, 1697-1701.

45 Y. Kim, Y. Jhon, J. Park, C. Kim, S. Lee and Y. Jhon, Sci. Rep., 2016, 6, 21405.

46 F. Li, Y. Yan, B. Han, L. Li, X. Huang, M. Yao, Y. Gong, X. Jin, B. Liu, C. Zhu, Q. Zhou and T. Cuia, Nanoscale, 2015, 7, 90759082.

47 G. Plechinger, F. X. Schrettenbrunner, J. Eroms, D. Weiss, C. Schuller and T. Korn, Phys. Status Solidi RRL, 2012, 6(3), 126-128.

48 C. Hu, X. Zeng, J. Cui, H. Chen and J. Lu, J. Phys. Chem. C, 2013, 117, 20998-21005. 